\title{
QCD sum rules: Isospin symmetry breakings in pion-nucleon couplings
}

\author{
W-Y. P. Hwang, ${ }^{1,2}$ Ze-sen Yang, ${ }^{3}$ Y. S. Zhong, ${ }^{3}$ Z. N. Zhou, ${ }^{3}$ and Shi-lin Zhu ${ }^{3}$ \\ ${ }^{1}$ Department of Physics, National Taiwan University, Taipei, Taiwan 10764 \\ ${ }^{2}$ Center for Theoretical Physics, Laboratory for Nuclear Science and Department of Physics, Massachusetts Institute of Technology, \\ Cambridge, Massachusetts 02139 \\ ${ }^{3}$ Department of Physics, Peking University, Beijing 100871, China \\ (Received 21 October 1996; revised manuscript received 8 September 1997)
}

\begin{abstract}
We use the method of QCD sum rules in the presence of an external point field to investigate isospin symmetry breakings in pion-nucleon couplings. Possible manifestations of such isospin symmetry breakings are examined in the context of low-energy nucleon-nucleon scattering. We discuss numerical results in relation to both the existing data and other theoretical predictions. [S0556-2813(98)04801-8]

PACS number(s): 24.80.+y, 21.30.-x, 13.75.Cs
\end{abstract}

\section{INTRODUCTION}

Isospin symmetry and its possible violations constitute an integral part of nuclear physics [1], not only in the sense that good symmetry offers an effective classification of nuclear or hadronic structure but also in the proximity of the isospin symmetry concept to other fundamental ideas such as chiral symmetry. In general, isospin symmetry breaking effects observed in nuclear physics may arise from different sources such as, in addition to the well known electromagnetic interactions, strong interactions but augmented with hadron mass differences (such as the $n-p$ or $\pi^{ \pm}-\pi^{0}$ mass difference) or with different meson-nucleon couplings (such as nonuniversal $\pi N N$ couplings) or with isospin mixings (such as $\rho-\omega$ or $\pi-\eta$ mixing). Determination of isospin symmetry breaking effects requires information on quantities which may not be directly accessible from experiments, such as the mesonnucleon couplings or the isospin mixing parameters. Accordingly, reliable theoretical predictions become indispensible for advancing our understanding of the problem.

Unfortunately, it appears that a quantitative treatment of isospin symmetry breakings may involve the capability of treating strong interactions at a quantitative level or, in other words, the ability to handle the complications due to quantum chromodynamics (QCD) in a reliable manner. To some extent, the method of QCD sum rules, as proposed originally by Shifman, Vainshtein, and Zakharov [2] and adopted, or extended, by many others [3-5], incorporates the nonperturbative QCD effects through various condensates (associated with the nontrivial QCD vacuum), thereby offering some hope of being able to treat strong interactions in a quantitative manner.

In this work, we wish to focus on the determination of isospin symmetry breakings in pion-nucleon couplings, with some emphasis on possible manifestations of such symmetry breaking in nucleon-nucleon scattering. As the present effort is based primarily on the method of QCD sum rules, it differs much from the quark-model calculations [6,7]; as we shall see, this work is considerably more extensive than a recent work [8] also making use of the QCD sum rule method. Specifically, we wish to adopt a method in which the pion field $\pi$ is treated as the external field. The idea of treating the pion as an external field was first suggested in [4] and has not been used in any extensive manner until recently $[9,10]$.

\section{ISOSPIN SYMMETRY BREAKINGS IN PION-NUCLEON COUPLINGS}

In the method of QCD sum rules in the presence of an external field, we attempt to evaluate, both at the quark and hadron levels, the correlation function specified by

$$
\left.\Pi(p) \equiv i \int d^{4} x e^{i p \cdot x}\left\langle 0\left|T\left[\eta_{p}(x) \bar{\eta}_{p}(0)\right]\right| 0\right\rangle\right|_{\pi},
$$

where we have

$$
\begin{aligned}
& \eta_{p}(x)=\epsilon^{a b c}\left\{u^{a T}(x) C \gamma_{\mu} u^{b}(x)\right\} \gamma^{5} \gamma^{\mu} d^{c}(x), \\
& \bar{\eta}_{p}(y)=\epsilon^{a b c}\left\{\bar{u}^{b}(y) \gamma_{\nu} C \bar{u}^{a T}(y)\right\} \bar{d}^{c}(y) \gamma^{\nu} \gamma^{5} .
\end{aligned}
$$

This correlation function allows us to determine the $\pi^{0} p p$ coupling. By evaluating the appropriate correlation function in the presence of the external pion field, we may determine the pion-nucleon couplings $g_{\pi^{0} p p}, g_{\pi^{0} n n}$, and $g_{\pi^{+} p n}$. It appears that the induced condensates involved in the external field method are now well understood and it becomes relatively straightforward to perform calculations to higher dimensions (as required for reliable predictions). This is what we choose to use in this paper and we use it to obtain isospin symmetry breakings in $\pi N N$ couplings.

At this juncture, we wish to note that an alternative method is to evaluate correlation functions with the $T$ product of currents sandwiched between the vacuum and the onepion state, as first suggested by [11] and recently adopted by some authors [12-14]. Using the soft-pion theorem, we may rewrite such matrix element, between the vacua, of the commutator of the axial charge $Q_{5}$ and the original $T$ product of the currents, thereby resulting in a $g_{\pi N N}$ sum rule which is related to the nucleon mass sum rules in some way. Unfortunately, a precise relation cannot be established unless the commutator between the axial charge and the nucleon current $\eta(x)$ is known. Note that the knowledge of such a commutator goes beyond current algebra and chiral dynamics. 
On the other hand, it is far from trivial if any such relation can be established in the external-field QCD sum rule method in which it is not necessary to take the soft-pion limit. An important indication as to why we may be away from the soft-pion limit is that the quark masses (and some other chiral symmetry breaking parameters) are not set to zero throughout the derivation. Indeed, the sum rule which we obtain in what follows for $g_{\pi N N}$ cannot be obtained from the nucleon mass sum rules by some chiral rotation and thus represents independent information. Nevertheless, it appears that such an aspect is fairly subtle and deserves further investigation.

At the quark level, we have

$$
\begin{aligned}
& \left\langle 0\left|T \eta_{p}(x) \bar{\eta}_{p}(0)\right| 0\right\rangle_{\pi^{0}} \\
& =2 i \epsilon^{a b c} \epsilon^{a^{\prime} b^{\prime} c^{\prime}} \operatorname{Tr}\left\{S_{u}^{b b^{\prime}}(x) \gamma_{\nu} C\left[S_{u}^{a a^{\prime}}(x)\right]^{T} C \gamma_{\mu}\right\} \\
& \quad \times \gamma_{5} \gamma_{\mu} S_{d}^{c c^{\prime}}(x) \gamma_{\nu} \gamma_{5} .
\end{aligned}
$$

Here the quark propagator is given, in configuration space, by

$$
S(x)=S^{(0)}(x)+S^{(\pi)}(x),
$$

with

$$
\begin{aligned}
i S^{(0) a b}(x)= & \frac{i \delta^{a b}}{2 \pi^{2} x^{4}} \hat{x}+\frac{i}{32 \pi^{2} x^{2}} \frac{\lambda_{a b}^{n}}{2} g_{c} G_{\mu \nu}^{n}\left(\sigma^{\mu \nu} \hat{x}+\hat{x} \sigma^{\mu \nu}\right) \\
& -\frac{\delta^{a b}}{12}\langle\overline{q q}\rangle+\frac{\delta^{a b} x^{2}}{192}\left\langle g_{c} \bar{q} \sigma \cdot G q\right\rangle-\frac{m_{q} \delta^{a b}}{4 \pi^{2} x^{2}} \\
& +\frac{m_{q}}{32 \pi^{2}} \frac{\lambda_{a b}^{n}}{2} G_{\mu \nu}^{n} \sigma^{\mu \nu} \ln \left(-x^{2}\right) \\
& -\frac{\delta^{a b}\left\langle g_{c}^{2} G^{2}\right\rangle}{2^{9} \times 3 \pi^{2}} m_{q} x^{2} \ln \left(-x^{2}\right)+\frac{i \delta^{a b} m_{q}}{48}\langle\overline{q q}\rangle \hat{x} \\
& -\frac{1}{2^{7} \times 3^{2}} \operatorname{im}_{q}\left\langle g_{c} \bar{q} \sigma \cdot G q\right\rangle \delta^{a b} x^{2},
\end{aligned}
$$

and

$$
\begin{aligned}
i S^{(\pi) a b}(x)= & -\frac{i \delta^{a b}}{4 \pi^{2} x^{2}} g_{q} \vec{\tau} \cdot \vec{\pi} \gamma_{5}+\frac{i \delta^{a b}}{24} g_{q} \vec{\tau} \cdot \vec{\pi} \gamma_{5} \chi\langle\overline{q q}\rangle \\
& -\frac{i \delta^{a b}}{384} m_{0}^{\pi} g_{q} \vec{\tau} \cdot \vec{\pi} \gamma_{5}\langle\overline{q q}\rangle x^{2} \\
& +\frac{i}{2^{5} \pi^{2}} g_{c} g_{q} \vec{\tau} \cdot \vec{\pi} \gamma_{5} \frac{\lambda_{a b}^{n}}{2} G_{\mu \nu}^{n} \sigma^{\mu \nu} \ln \left(-x^{2}\right) \\
& -\frac{i \delta^{a b}}{2^{9} \times 3 \pi^{2}} g_{q} \vec{\tau} \cdot \vec{\pi} \gamma_{5}\left\langle g_{c}^{2} G^{2}\right\rangle x^{2} \ln \left(-x^{2}\right) \\
& -\frac{\delta^{a b}}{48} g_{q} \vec{\tau} \cdot \vec{\pi} \gamma_{5}\langle\bar{q} q\rangle \hat{x}
\end{aligned}
$$

where $\hat{x} \equiv x_{\mu} \gamma^{\mu}$ and we have also introduced

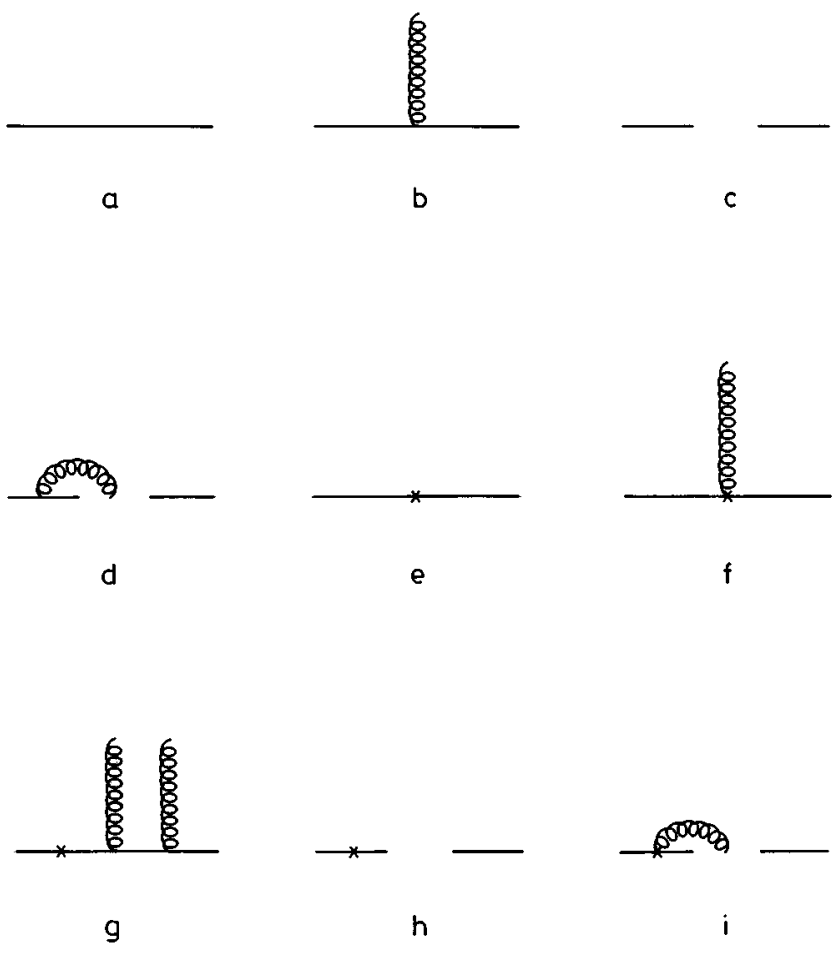

FIG. 1. Pictorial representation of the various terms in the quark propagator $S^{(0) a b}(x)$.

$$
\left\langle\bar{\psi}(0) i \gamma^{5} \tau^{j} \psi(0)\right\rangle \equiv g_{q} \chi \pi^{j}\langle\overline{q q}\rangle
$$

and

$$
\left\langle\bar{\psi}(0) i \gamma^{5} \tau^{j} g_{c} \sigma \cdot G \psi(0)\right\rangle \equiv g_{q} m_{0}^{\pi} \tau^{j}\langle\overline{q q}\rangle
$$

with $\psi$ the isospin doublet consisting of $u$ and $d$ quarks. The various terms in the quark propagators $i S^{(0) a b}(x)$ and $i S^{(\pi) a b}(x)$ may be represented pictorially by the diagrams shown in Figs. 1 and 2, respectively. We wish to stress that we have in fact performed our calculations in momentum space especially when ambiguities arise and, in addition, special attention has been directed to adoption of the $\gamma_{5}$ in $d$ dimensions in relation to dimensional regularization.
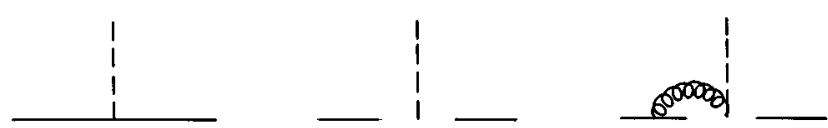

c

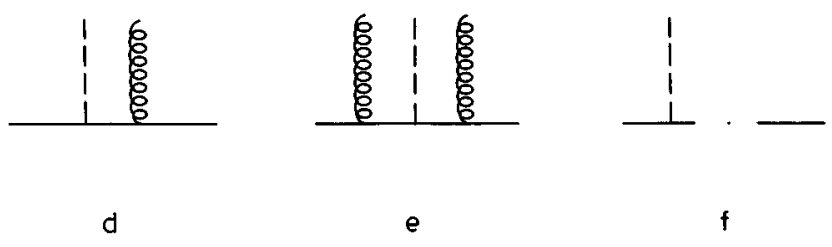

FIG. 2. Pictorial representation of the various terms in the quark propagator $S^{(\pi) a b}(x)$. 


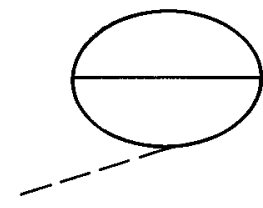

a

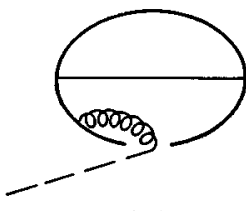

$d-1$

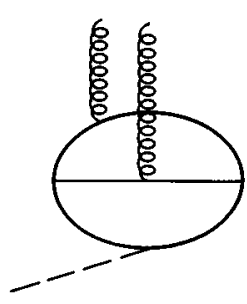

$f-1$

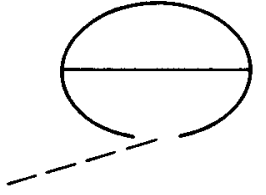

b
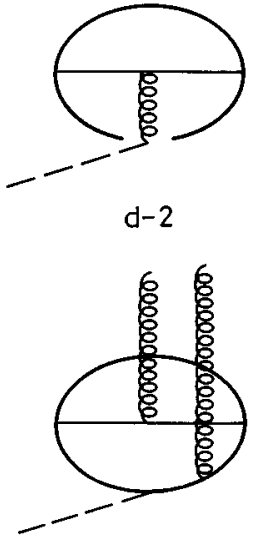

$f-2$

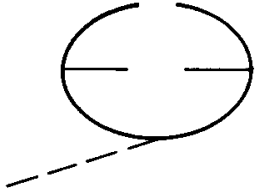

c
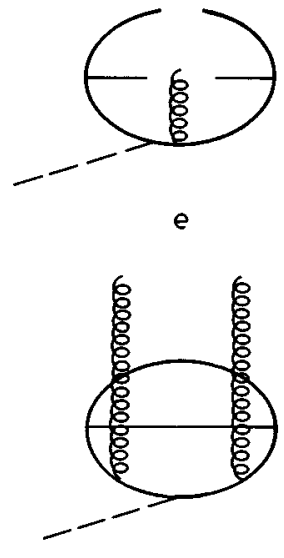

$f-3$

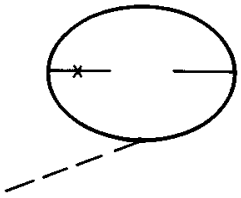

g-1

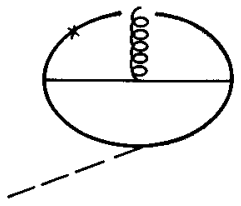

$h-2$

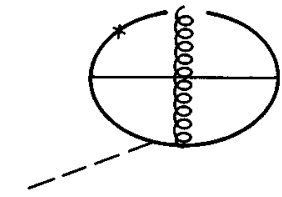

$h-3$

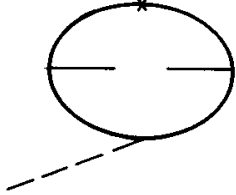

g-2

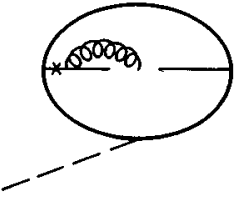

$h-1$

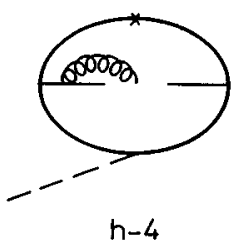

FIG. 3. The diagrams used to determine the QCD sum rule for $g_{\pi^{0} p p}$.

To incorporate isospin symmetry violations, we note that $\langle\bar{q} q\rangle$ appearing in the quark propagator needs to be interpreted properly; in particular, it is to be understood as $\langle\bar{u} u\rangle$ in the $(u u)$ channel, or as $\langle\bar{d} d\rangle$ in the $(d d)$ channel, or as $(\langle\bar{u} u\rangle+\langle\bar{d} d\rangle) / 2$ for $(d \rightarrow u)$ or $(u \rightarrow d)$ propagation. We also note that Fig. 2(f) has been drawn in a way to indicate that the choice of $q$ in $\langle\bar{q} q\rangle$ should follow the flavor of the quark connecting to $x=0$ (on the right-hand side of the diagram) - a result following the usage of the equation of motion. No potential ambiguity should arise for all the other diagrams shown in Figs. 1 and 2. The assumption has also been made that, once we have made the suitable interpretation of $\langle\overline{q q}\rangle, \chi$ and $m_{0}^{\pi}$ are considered to be universal. In other words, we refrain from introducing, at the quark level, further unknown sources for isospin symmetry violations; our assumption regarding the universal nature of the susceptibilities $\chi$ and $m_{0}^{\pi}$ is further supported by the derivation of induced condensates as illustrated in [10]. This is a crucial point since the terms in $\chi\langle\bar{u} u\rangle$ and $\chi\langle\bar{d} d\rangle$ turn out to be one of the most important contributions which distinguish the coupling $g_{\pi^{0} p p}$ from $g_{\pi^{0} n n}$.

We note that, for the determination of $\pi^{-} p n$ or $\pi^{+} n p$ coupling, we find

$$
\begin{aligned}
& \left\langle 0\left|T \eta_{p}(x) \bar{\eta}_{n}(0)\right| 0\right\rangle_{\pi^{-}} \\
& =-4 i \epsilon^{a b c} \epsilon^{a^{\prime} b^{\prime} c^{\prime}} \gamma_{\mu} \gamma_{5} S_{d}^{a a^{\prime}}(x) \gamma_{\nu} C\left[S^{(\pi) b b^{\prime}}(x)\right]^{T} \\
& \quad \times C \gamma_{\mu} S_{u}^{c c^{\prime}}(x) \gamma_{5} \gamma_{\nu} .
\end{aligned}
$$

We note that the absence of $i S^{(0) b b^{\prime}}(x)$ in the present case is due to a change in the electric charge. We also note that the structure (which does not involve the trace of some product) seems quite different from the $\pi^{0} p p$ case, but we will see that isospin symmetry is exact should we assume $m_{u}=m_{d}$ and $\langle\bar{u} u\rangle=\langle\bar{d} d\rangle$. Finally, to obtain QCD sum rules up to dimension eight (as compared to the leading perturbative term), we need to enumerate diagrams which are proportional to $1, \quad \chi\langle\bar{q} q\rangle,\left\langle g_{c}^{2} G^{2}\right\rangle, \quad m_{0}^{\pi}\langle\overline{q q}\rangle,\langle\bar{q} q\rangle^{2}$, $\langle\bar{q} q\rangle\left\langle g_{c} \bar{q} \sigma \cdot G q\right\rangle, m_{q}\langle\bar{q} q\rangle$, and $m_{q}\left\langle\bar{q} g_{c} \sigma \cdot G q\right\rangle$. Here it is clearly of importance to be able to distinguish between $\langle\bar{d} \hat{O} d\rangle$ and $\langle\bar{u} \hat{O} u\rangle$ in the quark propagators used to evaluate the correlation functions.

On the other hand, we may parametrize the correlation functions at the hadronic level in the standard manner:

$$
\begin{aligned}
& \left.\int d^{4} x\left\langle 0\left|T \eta_{p}(x) \bar{\eta}_{n}(0)\right| 0\right\rangle\right|_{\pi^{-}} \\
& =\lambda_{n} \lambda_{p} \frac{i}{\hat{p}-m_{p}}\left(-\gamma_{5} \sqrt{2} g_{\pi^{-} p n}\right) \frac{i}{\hat{p}-m_{n}}+\cdots, \\
& \left.\int d^{4} x\left\langle 0\left|T \eta_{p}(x) \bar{\eta}_{p}(0)\right| 0\right\rangle\right|_{\pi^{0}} \\
& =\lambda_{p}^{2} \frac{i}{\hat{p}-m_{p}}+\lambda_{p}^{2} \frac{i}{\hat{p}-m_{p}}\left(-\gamma_{5} g_{\pi^{0} p p}\right) \frac{i}{\hat{p}-m_{p}}+\cdots
\end{aligned}
$$

We consider the various diagrams shown in Fig. 3 and obtain the quark-level expression [referred to as the left-hand side (LHS)] for the $\pi^{0} p p$ coupling (directly from the momentumspace calculations), 


$$
\begin{aligned}
& \frac{1}{(2 \pi)^{4}} g_{\pi^{0} d d} \phi \gamma_{5}\left\{\frac{p^{4} \ln \left(-p^{2}\right)}{2}-\frac{\chi}{2} a_{d} p^{2} \ln \left(-p^{2}\right)\right. \\
& -\frac{11}{24} b \ln \left(-p^{2}\right)+\frac{4}{3} a_{u}^{2} \frac{1}{p^{2}}-\frac{1}{3} a_{u}^{2} m_{0}^{2} \frac{1}{p^{4}} \\
& +\frac{\ln \left(-p^{2}\right)+\gamma_{E}}{p^{2}} m_{0}^{2}\left[\frac{7}{8}\left(a_{u} m_{d}+a_{d} m_{u}\right)-\frac{1}{4} m_{u} a_{u}\right] \\
& \left.+\frac{m_{0}^{2}}{p^{2}}\left[\frac{5}{12} m_{u} a_{u}-\frac{1}{8} m_{d} a_{d}+\frac{67}{48} m_{d} a_{u}+\frac{115}{48} m_{u} a_{d}\right]\right\}
\end{aligned}
$$

with $a_{q} \equiv-(2 \pi)^{2}\langle\overline{q q}\rangle,\langle\bar{q} \sigma \cdot G q\rangle \equiv-m_{0}^{2}\langle\bar{q} q\rangle$, and $b$ $\equiv\left\langle g_{c}^{2} G^{2}\right\rangle$. Accordingly, we obtain, upon Borel transform, the following QCD sum rule for the $\pi^{0} p p$ coupling:

$$
\begin{aligned}
M^{6}- & \frac{\chi}{2} a_{d} M^{4}-\frac{11}{24} b M^{2}+\frac{4}{3} a_{u}^{2}+\frac{m_{0}^{2}}{3} a_{u}^{2} \frac{1}{M^{2}} \\
& +m_{0}^{2} \ln \frac{M^{2}}{\mu^{2}}\left[\frac{7}{8}\left(a_{u} m_{d}+a_{d} m_{u}\right)-\frac{1}{4} m_{u} a_{u}\right] \\
& +m_{0}^{2}\left[\frac{5}{12} m_{u} a_{u}-\frac{1}{8} m_{d} a_{d}+\frac{67}{48} m_{d} a_{u}+\frac{115}{48} m_{u} a_{d}\right] \\
= & \frac{g_{\pi^{0} p p}}{g_{\pi^{0} d d}} \widetilde{\lambda}_{p}^{2} e^{-m_{p}^{2} / M^{2}}+\text { excited states continuum, }
\end{aligned}
$$

where $M$ is the Borel mass and $\widetilde{\lambda}_{p}^{2}=(2 \pi)^{4} \lambda_{p}^{2}$. A similar expression can be obtained for the $\pi^{0} n n$ coupling through the replacement $u \leftrightarrow d$. It is of some interest to note that the contribution from Figs. 3(d1) is cancelled by that from Fig. $3(\mathrm{~d} 2)$, so that the induced susceptibility $m_{0}^{\pi}$ does not enter in the determination of strong $\pi N N$ coupling, an aspect already observed in [9].

For the $\pi^{+} n p$ coupling, we have, with $\bar{a} \equiv\left(a_{u}+a_{d}\right)$,

$$
\begin{aligned}
& M^{6}-\frac{\chi}{2} \bar{a} M^{4}-\frac{11}{24} b M^{2}+\frac{4}{3} a_{u} a_{d}+\frac{1}{3}\left(a_{d}-a_{u}\right) a_{d}-\left(m_{u}\right. \\
& \left.-m_{d}\right) a_{u} M^{2}+m_{0}^{2}\left\{-\frac{1}{2} a_{u} m_{u}-\frac{5}{24} a_{d} m_{d}+\frac{29}{12} a_{u} m_{d}\right. \\
& \left.+\frac{19}{8} a_{d} m_{u}\right\}+m_{0}^{2} \ln \frac{M^{2}}{\mu^{2}}\left\{a_{u} m_{d}+a_{d} m_{u}-\frac{1}{4} m_{u}\left(a_{d}\right.\right. \\
& \left.\left.+a_{u}\right)\right\}+\frac{m_{0}^{2}}{3} a_{u} a_{d} \frac{1}{M^{2}}=\frac{g_{\pi^{+} n p}}{g_{\pi^{+}} d u}(2 \pi)^{4} \lambda_{n} \lambda_{p} e^{-m^{2} / M^{2}} \\
& + \text { excited states + continuum. }
\end{aligned}
$$

As a standard practice, we may improve the applicability of these QCD sum rules by (i) taking into account the anomalous dimension of each term (operator) and (ii) invoking the continuum approximation in which contributions from excited states and the continuum are approximated by what we may obtain at the quark level above a certain threshold $W$.
Results from such improvements can be duplicated easily from Yang et al. [15]. In this way, we obtain, for the $\pi^{0} p p$ coupling [15,9],

$$
\begin{aligned}
M^{6} L^{-4 / 9} E_{2}-\frac{\chi}{2} a_{d} M^{4} L^{2 / 9} E_{1}-\frac{11}{24} b M^{2} E_{0}+\frac{4}{3} a_{u}^{2} L^{4 / 9} \\
+\frac{m_{0}^{2}}{3} a_{u}^{2} \frac{1}{M^{2}} L^{-2 / 27}+m_{0}^{2} \ln \frac{M^{2}}{\mu^{2}} L^{-26 / 27}\left[\frac { 7 } { 8 } \left(a_{u} m_{d}\right.\right. \\
\left.\left.+a_{d} m_{u}\right)-\frac{1}{4} m_{u} a_{u}\right]+m_{0}^{2} L^{-26 / 27}\left[\frac{5}{12} m_{u} a_{u}-\frac{1}{8} m_{d} a_{d}\right. \\
\left.+\frac{67}{48} m_{d} a_{u}+\frac{115}{48} m_{u} a_{d}\right]=\frac{g_{\pi^{0} p p}}{g_{\pi^{0} d d}} \widetilde{\lambda}_{p}^{2} e^{-m_{p}^{2} / M^{2}},
\end{aligned}
$$

and, for the $\pi^{+} n p$ coupling,

$$
\begin{gathered}
M^{6} L^{-4 / 9} E_{2}-\frac{\chi}{2} \bar{a} M^{4} L^{2 / 9}-\frac{11}{24} b M^{2} E_{0}+\left\{\frac{4}{3} a_{u} a_{d}+\frac{1}{3}\left(a_{d}\right.\right. \\
\left.\left.-a_{u}\right) a_{d}\right\} L^{4 / 9}-\left(m_{u}-m_{d}\right) a_{u} M^{2} L^{-4 / 9} E_{0}+m_{0}^{2} L^{-26 / 27} \\
\quad \times\left\{-\frac{1}{2} a_{u} m_{u}-\frac{5}{24} a_{d} m_{d}+\frac{29}{12} a_{u} m_{d}+\frac{19}{8} a_{d} m_{u}\right\} \\
+m_{0}^{2} \ln \frac{M^{2}}{\mu^{2}} L^{-26 / 27}\left\{a_{u} m_{d}+a_{d} m_{u}-\frac{1}{4} m_{u}\left(a_{d}+a_{u}\right)\right\} \\
+\frac{m_{0}^{2}}{3} a_{u} a_{d} L^{-2 / 27} \frac{1}{M^{2}}=\frac{g_{\pi^{+} n p}}{g_{\pi^{+} d u}}(2 \pi)^{4} \lambda_{n} \lambda_{p} e^{-m^{2} / M^{2}}
\end{gathered}
$$

Here $E_{0}=1-e^{-x}, E_{1}=1-(1+x) e^{-x}$, and $E_{2}=1-(1+x$ $\left.+x^{2} / 2\right) e^{-x}$ with $x=W / M^{2}$, and $L=0.621 \ln (10 M)$.

There are several sources for isospin symmetry breakings, viz., at the hadronic level (RHS), we have $m_{n} \neq m_{p}, \lambda_{n}$ $\neq \lambda_{p}$, and $W_{n} \neq W_{p}$ (with $W$ the threshold parameter in the continuum approximation for treating the excited states and the continuum). Apart from the well known neutron-proton mass difference, we use the parameters previously adopted by Yang et al. [15].

At the quark level (LHS), the isospin symmetry breaking caused by $\langle\bar{d} d\rangle \neq\langle\bar{u} u\rangle$ and $m_{d} \neq m_{u}$ have been made explicit in the formulas given above. Such effects have also been considered by other authors $[8,15]$.

In addition, there is an effect due to the non-universal pion-quark couplings. Conceptually, we may start with a universal pion-quark coupling, such as in the effective chiral quark theory, but the vertex renormalizations due to electromagnetic interactions, as shown in Fig. 4 and also pointed out by Cao and Hwang [6], modify $\pi^{0} u u, \pi^{0} d d$, and $\pi^{+} u d$ in a different manner. Here we have adopted the dimensional regularization scheme to work out such differences and conclude that the nonuniversal pion-quark couplings so obtained could be a reasonably important source for isospin symmetry 

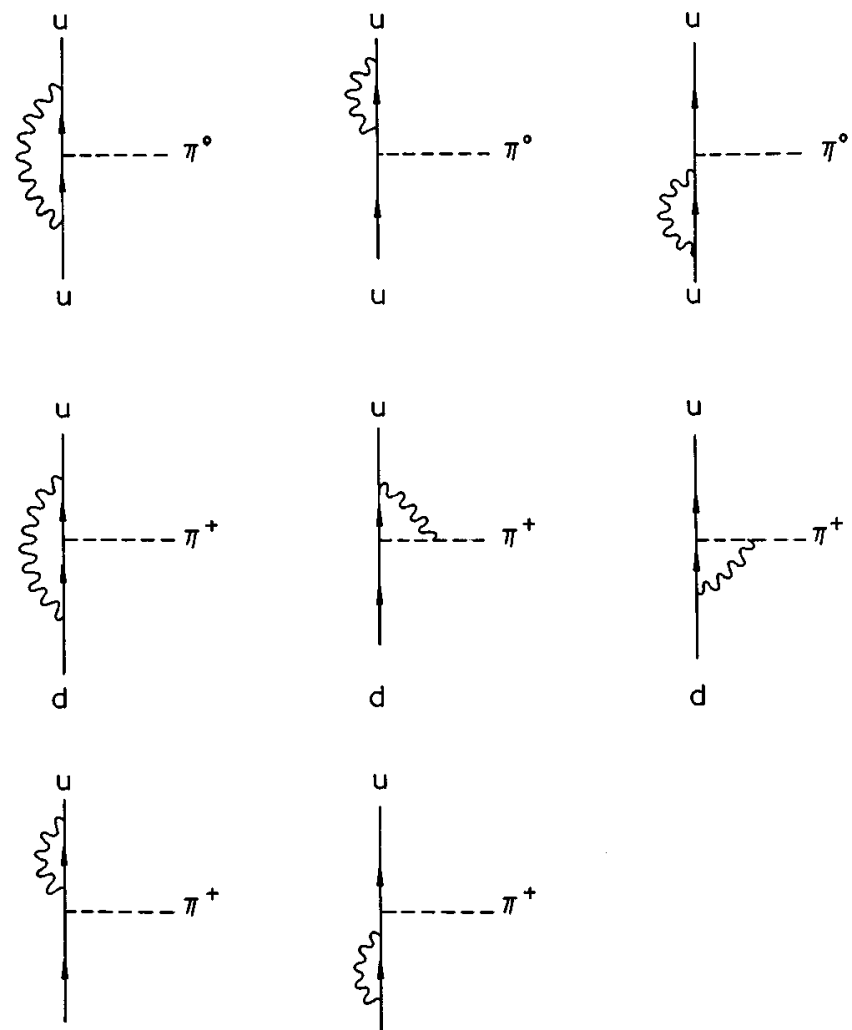

d

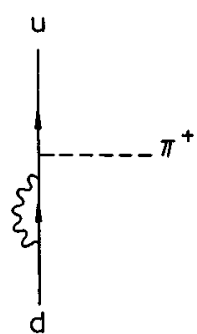

FIG. 4. The vertex renormalizations (including the self-energy contributions) leading to the nonuniversal pion-quark couplings, an important source for isospin symmetry breakings.

breaking. Specifically, we obtain, in the minimum subtraction scheme, the following expressions for the pion-quark couplings:

$$
\begin{gathered}
g_{\pi^{+} d u} \rightarrow g_{\pi^{+} d u}\left\{1+\frac{\alpha}{4 \pi}\left(-\frac{43}{18}+\frac{1}{6} \gamma_{E}\right)\right\}, \\
g_{\pi^{0} u u} \rightarrow g_{\pi^{0} u u}\left\{1+\frac{\alpha}{4 \pi}\left(\frac{52}{9}-\frac{4}{3} \gamma_{E}\right)\right\}, \\
g_{\pi^{0} d d} \rightarrow g_{\pi^{0} d d}\left\{1+\frac{\alpha}{4 \pi}\left(\frac{13}{9}-\frac{1}{3} \gamma_{E}\right)\right\} .
\end{gathered}
$$

Numerically, these expressions give rise to $2.18 \times 10^{-3}$ to the charge symmetry breaking term $g_{\pi^{0} u u}-g_{\pi^{0} d d}$ and 4.2 $\times 10^{-3}$ for the charge dependent combination $g_{\pi^{0} u u}$ $-g_{\pi^{+} d u}$.

To perform numerical analyses, we introduce the following parameters:

$$
\begin{gathered}
\lambda \equiv \frac{\lambda_{p}+\lambda_{n}}{2}, \quad \sigma \equiv \frac{\lambda_{n}-\lambda_{p}}{\lambda} \\
\bar{m} \equiv \frac{m_{p}+m_{n}}{2}, \quad \epsilon \equiv \frac{m_{n}-m_{p}}{\bar{m}},
\end{gathered}
$$

$$
\begin{gathered}
\frac{g_{\pi^{0} p p}}{g_{\pi^{0} d d}} \equiv K, \\
\frac{g_{\pi^{0} n n}}{g_{\pi^{0} u u}} \equiv K\left(1+\rho_{1}\right), \\
\frac{g_{\pi^{-} p n}}{g_{\pi^{-} u d}} \equiv K\left(1+\rho_{2}\right), \\
\frac{g_{\pi^{+} n p}}{g_{\pi^{+} d u}} \equiv K\left(1+\rho_{3}\right) .
\end{gathered}
$$

Moving the pion-quark coupling to the phenomenological side, we may write the RHS as follows:

$$
\begin{gathered}
\pi^{0} p p \rightarrow K \lambda^{2} e^{-m^{2} / M^{2}}\left(1-\sigma+\epsilon \frac{m^{2}}{M^{2}}\right), \\
\pi^{0} n n \rightarrow K \lambda^{2} e^{-m^{2} / M^{2}}\left(1+\sigma-\epsilon \frac{m^{2}}{M^{2}}+\rho_{1}\right), \\
\pi^{-} p n \rightarrow K \lambda^{2} e^{-m^{2} / M^{2}}\left(1+\rho_{2}\right), \\
\pi^{+} n p \rightarrow K \lambda^{2} e^{-m^{2} / M^{2}}\left(1+\rho_{3}\right) .
\end{gathered}
$$

The various parameters which we adopt are $\sigma=-10^{-3}$ [15], $\epsilon=1.3 \times 10^{-3}, \gamma=-6.57 \times 10^{-3}[16], a_{q}=0.546 \mathrm{GeV}^{3}, m_{u}$ $=5.1 \mathrm{MeV}, m_{d}=8.9 \mathrm{MeV}, b=0.474 \mathrm{GeV}^{4}, m_{0}^{2}=0.8 \mathrm{GeV}^{2}$, $\mu=0.5 \mathrm{GeV}$, and $\chi a=-3.14 \mathrm{GeV}^{2}[10]$. This set of parameter values is to be referred to as the "standard set"'in the rest of this paper. The working interval for analyzing the QCD sum rules for the various $\pi N N$ couplings is $0.8 \mathrm{GeV}^{2} \leqslant M_{B}^{2} \leqslant 1.5 \mathrm{GeV}^{2}$, a standard choice for analyzing the various QCD sum rules associated with the nucleon.

The QCD sum rules (13) and (14) yield a prediction on the strong $\pi N N$ coupling, which is numerically quite stable (as compared to, e.g., the nucleon mass sum rules). Figures $5(a)-5(c)$ display such numerical stability for a broad range of parameter values. In Fig. 5(a), the predicted strong $\pi N N$ coupling is shown as a function of the Borel mass squared $M_{B}^{2}$ (in units of $\mathrm{GeV}^{2}$ ) for three different values of the continuum threshold $W$. Note that in [15] a standard value of $W=2.25 \mathrm{GeV}^{2}$ on the continuum threshold is adopted to ensure numerical stability of in the working interval $0.8 \mathrm{GeV}^{2}$ $\leqslant M_{B}^{2} \leqslant 1.5 \mathrm{GeV}^{2}$. In Fig. 5(b), similar plots are given for three different values of the quark condensate $a$, while in Fig. 5(c) the sensitivity of the sum rule to the susceptibility $\chi$ is illustrated.

Figures 5(a)-5(c) indicate that the QCD sum rule for the strong $\pi N N$ coupling, Eqs. (13) and (14), is numerically well behaved and may be used with some confidence. It yields a numerical prediction of $g_{\pi N N}=-(13.5 \pm 1.5)$, with the error estimated by assuming $\Delta W=0.05 \mathrm{GeV}^{2}, \Delta a$ $=0.05 \mathrm{GeV}^{3}$, and $\Delta \chi=1.0 \mathrm{GeV}^{-1}$ and adding the errors quadratically. In analyzing the sum rule (13) or (14), it would also be useful to keep in mind that $g_{\pi N N}$ on the righthand side could be replaced by $g_{\pi N N}+B M^{2}$ in order to take into account the strength of the transition from, e.g., the continuum to the ground state, a contribution which may not be 

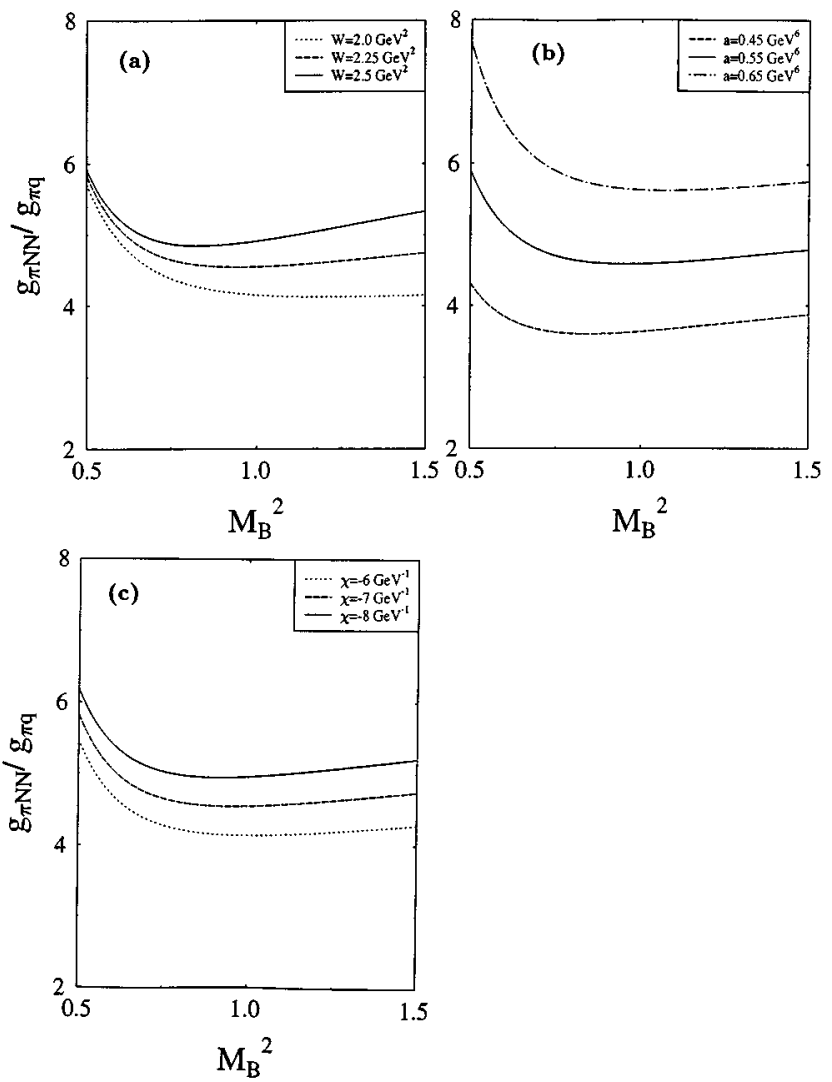

FIG. 5. $g_{\pi} N N / g_{\pi q}$ shown as a function of the Borel mass squared $M_{B}^{2}$ (in units of $\mathrm{GeV}^{2}$ ): (a) for three different values of the continuum threshold; (b) for three different values of the quark condensate; and (c) for three different values of the induced condensate as characterized by the susceptibility $\chi$.

sufficiently suppressed when the $\gamma_{5} \pi N N$ coupling is adopted. As already shown in [10], however, the resultant $\pi N N$ does not differ much from the value quoted above and the cited error is more than adequate to also cover such uncertainty.

Adopting the standard set of parameter values (as listed earlier), we then find

$$
\begin{gathered}
\frac{g_{\pi^{0} n n} / g_{\pi^{0} u u}-g_{\pi^{0} p p} / g_{\pi^{0} d d}}{g_{\pi^{0} N N} /\left.g_{\pi^{0} Q Q}\right|_{\text {average }}}=0.58 \%, \\
\frac{\left(g_{\pi^{+}{ }_{n p}}\right) /\left(g_{\pi^{+} d u}\right)-g_{\pi^{0} p p} / g_{\pi^{0} d d}}{g_{\pi^{0} N N} /\left.g_{\pi^{0} Q Q}\right|_{\text {average }}}=0.35 \% .
\end{gathered}
$$

Combining with the difference in pion-quark couplings (from vertex renormalizations), we therefore conclude that $g_{\pi^{0} n n}$ is numerically bigger than $g_{\pi^{0} p p}$ by $0.80 \%$, while $g_{\pi^{+} n p}$ is numerically greater than $g_{\pi^{0} p p}$ by $0.15 \%$.

Numerically, we may arbitrarily set $m_{d}=m_{u},\langle\bar{d} d\rangle$ $=\langle\bar{u} u\rangle$, and $\lambda_{n}=\lambda_{p}$, except keeping physical values for $m_{n}$

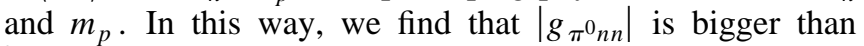
$\left|g_{\pi^{0} p p}\right|$ by $0.19 \%$. Analogously, we find that $\lambda_{n} \neq \lambda_{p}$ (as found by Yang et al. [15]) has a contribution of $0.20 \% . m_{d}$ $\neq m_{u}$ and $\langle\bar{d} d\rangle \neq\langle\bar{u} u\rangle$ combine to give another contribution of $0.20 \%$. Therefore, the overall contribution of $80 \%$ in fact comes from several sources of comparable magnitudes (and of the same sign in the present case).
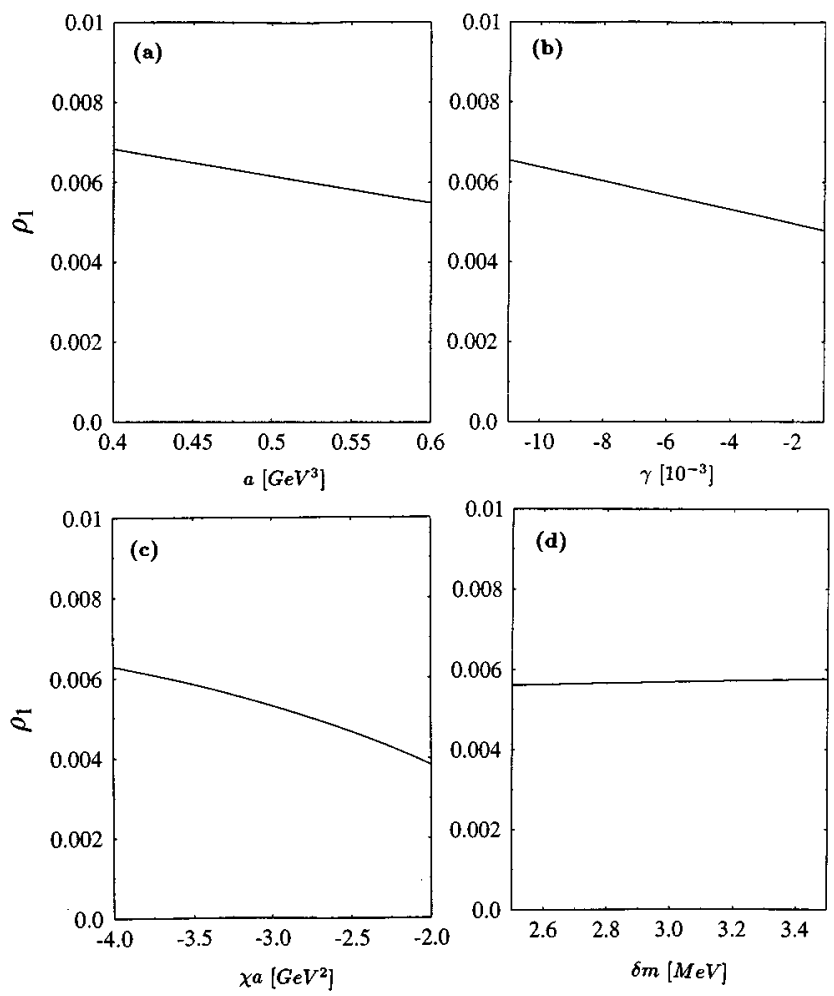

FIG. 6. The parameter $\rho_{1}$, which is essentially the left-hand side of Eq. (16), shown as a function of the given parameter while keeping all the other parameters fixed as standard values listed in the text: (a) by varying the quark condensate $a$, (b) by varying $\gamma$ (which characterizes the difference between the up and down quark condensates), (c) by varying the susceptibility, and (d) by varying $\delta m\left(\equiv m_{d}-m_{u}\right)$.

It is of some interest to note that our prediction on $\left|g_{\pi^{0} n n}\right|-\left|g_{\pi^{0} p p}\right|$ (a positive value) differs in sign from the quark-model result [6], and also from a three-point correlation function approach [8]. It is also of interest to note that the difference in pion-quark couplings represents a source of numerical importance on the charge symmetry breaking effect.

It is of some importance to assess the error in our prediction on the charge-symmetry breaking effect as characterized by $\left|g_{\pi^{0} n n}\right|-\left|g_{\pi^{0} p p}\right|$. To analyze this question, it is helpful to keep in mind that, although the nucleon mass sum rule may yield a prediction on the nucleon mass with an error as large as $(10-15) \%$, we are still able to make prediction on the nucleon-proton mass difference (which is only $0.1 \%$ of the average nucleon mass) and have a prediction which is also of $(10-20) \%$ in error [15]. The reason is simply that when we combine the sum rules for the proton and the neutron we may obtain another sum rule characterizing the differenceand such a sum rule is to be analyzed in its own light. The same situation occurs for the strong $\pi N N$ couplings, where we have combined the sum rules for $g_{\pi^{0} n n}$ and $g_{\pi^{0} p p}$ to obtain a sum rule for the difference and this is what is behind the predictions cited for Eqs. (16) and (17).

Numerically, we may adopt the standard set of parameter values but choose to vary one particular parameter in order to estimate the error. In Figs. 6(a)-6(d), we display the sample results. In Fig. 6(a), the sensitivity of $\rho_{1}$, which determines $g_{\pi^{0} n n} / g_{\pi^{0} u u}-g_{\pi^{0} p p} / g_{\pi^{0} d d}$, to the quark condensate $a$ is 
shown (while keeping $\gamma, \chi, \delta m$, and other parameters fixed at the standard values). A variation of $\pm 0.05 \mathrm{GeV}^{3}$ for $a$ yields an error on $\rho_{1}$ of less than $0.05 \%$. Analogously, we show in Figs. 6(b), 6(c), and 6(d), respectively, the sensitivity of $\rho_{1}$ to a reasonable variation in the parameters $\gamma$ (in units of $\left.10^{-3}\right), \chi a$ (in units of $\left.\mathrm{GeV}^{2}\right)$, and $\delta m\left(\equiv m_{d}-m_{u}\right.$, in units of MeV). Contrary to what has been found [6] in the naive quark model, the variation in $\delta m$ does not produce visible change in $\rho_{1}-$ a nontrivial aspect which manifests itself through the QCD sum rule. The variation of $\rho_{1}$ with respect to $\gamma$ is much milder than that of the neutron-proton mass difference to $\gamma$. In fact, the change in $\gamma$ is fairly restricted (i.e., of the order of $0.5 \times 10^{-3}$ to ensure that the prediction on $m_{n}-m_{p}$ has an error of about $20 \%$ ) if we wish to retain reasonable predictions on the neutron-proton mass difference. Even if we take the liberty to give a sizable uncertainty to $\gamma$, say as large as $4 \times 10^{-3}$, Fig. 6(b) suggests that the error on the prediction (16) would remain to be about $0.05 \%$. Finally, we caution that the sensitivity of $\rho_{1}$ to $a$ and $\chi$ as shown by Figs. 6(a) and 6(c) is caused by the nonzero value of $\gamma$ (which breaks charge symmetry), rather than by $a$ or $\chi$ itself-we have produced these figures only for the sake of estimating the errors.

To sum up, Figs. 6(a)-6(d) suggest that we may place an overall error of about $0.10 \%$ on our prediction in Eq. (16). Considering the different sources for the overall $0.58 \%$ as given by Eq. (16), such error estimate appears to be rather conservative since most of the sources actually do not involve any significant error and the $0.10 \%$ error estimated above arises primarily from possible error to a source of about $0.20 \%$.

As a parenthetical remark, we note that the situation is very different in the three-point correlation function approach [8], in which the pion-nucleon couplings are determined predominantly by the quark condensate and as a result the uncertainty in $\gamma$ translates itself directly into the error in $g_{\pi^{0} p p}-g_{\pi^{0} n n}$. In our case, the contribution from $\gamma$ is only some part of the whole story and a large variation of $\gamma$ does not cause as seriously a problem as in the three-point method. Nevertheless, it is amusing that different sum rules for the pion-nucleon coupling can be obtained based on different approaches and it is of some importance to pin down in future studies possible connections among these different sum rules.

\section{LOW-ENERGY NUCLEON-NUCLEON SCATTERING}

The one-pion-exchange potential (OPEP) in nucleonnucleon interactions can be obtained in the standard manner [6]. For example, we have, in the $p-p$ case,

$$
V_{p p}^{\pi}(r)=\frac{g_{\pi^{0} p p}^{2}}{4 \pi} \frac{\vec{\sigma}^{1} \cdot \vec{\nabla}_{r} \vec{\sigma}^{2} \cdot \vec{\nabla}_{r}}{4 m_{p}^{2}} \frac{1}{r}\left(e^{-m_{\pi^{0}}{ }^{r}}-e^{-\Lambda r}\right),
$$

where $\Lambda$ is the cutoff at the short range which contributes negligibly to the isospin symmetry breakings [6]. The OPEP in the $n-n$ case may be obtained by simple substitution $p \rightarrow n$. On the other hand, the $n-p$ OPE potential is given by

$$
\begin{aligned}
V_{n p}^{\pi}(r)= & \frac{g_{\pi^{0} n n} g_{\pi^{0} p p}}{4 \pi} \tau_{3}^{1} \tau_{3}^{2} \frac{\vec{\sigma}^{1} \cdot \vec{\nabla}_{r} \vec{\sigma}^{2} \cdot \vec{\nabla}_{r}}{4 m_{n} m_{p}} \frac{1}{r}\left(e^{-m_{\pi^{0}} r}-e^{-\Lambda r}\right) \\
& +\frac{g_{\pi^{+} n p}^{2}}{4 \pi}\left\{\left(\vec{\tau}^{1} \cdot \vec{\tau}^{2}-\tau_{3}^{1} \tau_{3}^{2}\right) \frac{\vec{\sigma}^{1} \cdot \vec{\nabla}_{r} \vec{\sigma}^{2} \cdot \vec{\nabla}_{r}}{4 M_{N}^{2}}\right. \\
& \left.+\frac{2 \delta}{4 M_{N}^{2}}\left(\vec{\tau}^{1} \times \vec{\tau}^{2}\right)_{3} \vec{\sigma}^{1} \times \vec{\sigma}^{2} \cdot \vec{L} \frac{1}{r} \frac{d}{d r}\right\} \frac{1}{r} \\
& \times\left(e^{-m_{\pi}+r}-e^{-\Lambda r}\right) .
\end{aligned}
$$

Here we have used $\delta \equiv\left(m_{n}-m_{p}\right) /\left(m_{n}+m_{p}\right)$ and $M_{N}$ $=\frac{1}{2}\left(m_{n}+m_{p}\right)$. The ${ }^{1} S_{0}$ phase shift $\delta_{0}$, as used to describe low-energy nucleon-nucleon scatterings, can be parametrized in the standard manner:

$$
k \cot \delta_{0}=-\frac{1}{a}+\frac{1}{2} k^{2} r_{0}+\cdots,
$$

with $a$ the scattering length and $r_{0}$ the effective range. Experimentally, we have [17]

$$
\begin{gathered}
\delta a_{\mathrm{CSB}} \equiv\left|a_{p p}\right|-\left|a_{n n}\right|=-(1.5 \pm 0.5) \mathrm{fm}, \\
\delta a_{\mathrm{CD}} \equiv\left|a_{n p}\right|-\left|a_{n n}\right|=(5.0 \pm 0.3) \mathrm{fm} .
\end{gathered}
$$

As already noted in [6], the differences in the OPEP's, such as $V_{p p}(r)-V_{n n}(r)$, may be treated as a perturbation, making it easy to compute $\delta a_{\mathrm{CSB}}$ or other low-energy isospin symmetry breaking observables. In this way, our prediction on the difference between $g_{\pi^{0} n n}$ and $g_{\pi^{0} p p}$ gives rise to about $-1.0 \mathrm{fm}$ on $\delta a_{\mathrm{CSB}}$, consistent with the observation both in sign and roughly in magnitude.

As has been known $[1,6]$ for some time, the bulk of the charge-dependent effect as revealed by a (relatively) large value of $\delta a_{\mathrm{CD}}$ can be understood quantitatively as caused by the pion mass difference $m_{\pi^{+}} \neq m_{\pi^{0}}$ while the differences as found in pion-nucleon couplings give rise to relatively small contribution to $\delta a_{\mathrm{CD}}$. As a result, we may safely conclude that the observed $\delta a_{\mathrm{CSB}}$ and $\delta a_{\mathrm{CD}}$ as summarized recently in [17] can be understood, to a large extent, as manifestations of isospin asymmetries associated with the one-pion exchange potential.

\section{DISCUSSION AND SUMMARY}

The observed differences [17] in the ${ }^{1} S_{0} N N$ scatterings are by far the best known isospin symmetry breakings in which pion-nucleon couplings play a central role. There might be some isospin symmetry breakings in the ${ }^{1} S_{0}$ effective ranges or in the ${ }^{3} P_{J}$ scattering lengths, but until now such effects have not been extracted in any reliable manner.

The last term in $V_{n p}^{\pi}(r)$ [in Eq. (19)], as induced by $m_{p}$ $\neq m_{n}$, gives rise to isospin mixings such as ${ }^{3} P_{1}-{ }^{1} P_{1}$ or ${ }^{3} D_{2}-{ }^{1} D_{2}$ mixing, but only in a rather small way. On the other hand, charge-symmetry breakings observed in medium-energy elastic $n-p$ scattering (at TRIUMF and IUCF) may be dictated primarily [7] by the charge symmetry breaking force as caused by the $\rho-\omega$ mixing or by $g_{\omega p p}$ $\neq g_{\omega n n}$. However, the short-range nature in this case may require a careful investigation of the quark-interchange 
mechanisms as addressed in [18], as it is difficult to reconcile the picture of exchanging, between two nucleons, a meson $(\rho$ or $\omega)$ with a range of about 0.25 fermi, considerably smaller than the extent of a nucleon (with a radius of at least 0.5 fermi, even assuming a little bag picture for the nucleon).

In any event, the isospin symmetry breakings associated with the one-pion exchange potential (which is clearly on a very sound conceptual ground) can best be accessed through careful measurements of low-energy scattering parameters such as $\delta a_{\mathrm{CSB}}, \delta a_{\mathrm{CD}}$, etc. Such efforts should be further encouraged by the currently popular wisdom that the lowenergy sector of the $\pi N$ are $N N$ systems can be described by the long -wave length limit of QCD via Goldstone theorem, yielding the so-called chiral perturbation theory, so that the low-energy scattering parameters seems to be more "fundamental" than those needed in, e.g., the description of medium-energy nucleon-nucleon scatterings.

In summary, we have adopted the method of QCD sum rules in the presence of an external pion field to investigate isospin symmetry breakings in pion- nucleon couplings. The resultant QCD sum rule for the strong pion-nucleon coupling is found to be well behaved and the prediction on $g_{\pi N N}$ is
$-(13.5 \pm 1.5)$ in good agreement with nuclear force studies. The QCD sum rule for the difference between $g_{\pi^{0} n n}$ and $g_{\pi^{0} p p}$ can be analyzed in its own light and the most uncertain part (which is caused directly or indirectly by $\gamma$ ) is by itself fairly small, thereby resulting in a small overall error. $\mathrm{Nu}-$ merically, we find that $\left|g_{\pi^{0} n n}\right|$ is bigger than $\left|g_{\pi^{0} p p}\right|$ by $(0.80 \pm 0.10) \%$ (and bigger than $\left|g_{\pi^{+}{ }_{n p}}\right|$ by $0.65 \%$ ), with its major portion determined reliably without significant error. This result accounts for the observed charge-symmetry breaking effect $\delta a_{\mathrm{CSB}}$ both in sign and (roughly) in magnitude. Contrary to many previous calculations in the literature, our result offers, for the first time, the possibility to understand both $\delta a_{\mathrm{CSB}}$ and $\delta a_{\mathrm{CD}}$ in a quantitative manner (both in sign and in magnitude).

\section{ACKNOWLEDGMENTS}

The authors wish to acknowledge Ernest M. Henley and Susan Gardner for helpful discussions. This work was supported in part by the National Science Council of the ROC (Grant No. NSC86-2112-M002-010Y).
[1] E. M. Henley and G. A. Miller, in Mesons in Nuclei, edited by M. Rho and D. H. Wilkinson (North-Holland, Amsterdam, 1979).

[2] M. A. Shifman, A. I. Vainshtein, and V. J. Zakharov, Nucl. Phys. B147, 385 (1979); B147, 448 (1979); B147, 519 (1979).

[3] L. I. Reinders, H. R. Rubinstein, and S. Yazaki, Nucl. Phys. B196, 125 (1982); L. I. Reinders, H. R. Rubinstein, and S. Yazaki, Phys. Rep. 127, 1 (1985).

[4] B. L. Ioffe, Nucl. Phys. B188, 317 (1981); B191, 591(E) (1981); V. M. Belyaev and B. L. Ioffe, Zh. Eksp. Teor. Fiz. 83, 876 (1982) [Sov. Phys. JETP 56, 493 (1982)]; B. L. Ioffe and A. V. Smilga, Phys. Lett. 114B, 353 (1982); Nucl. Phys. B232, 109 (1984).

[5] I. I. Balitsky and A. V. Yung, Phys. Lett. 129B, 328 (1983).

[6] Z.-J. Cao and W-Y. P. Hwang, Phys. Rev. C 29, 1 (1984).

[7] S. Gardner, C. J. Horowitz, and J. Piekarewicz, Phys. Rev. C 53, 1143 (1996).
[8] E. M. Henley and T. Meissner, Phys. Rev. C 55, 3093 (1997).

[9] E. M. Henley, W-Y. P. Hwang, and L. S. Kisslinger, Phys. Lett. B 367, 21 (1996).

[10] W-Y. P. Hwang, Z. Phys. C 75, 701 (1997).

[11] N. S. Craigie and J. Stern, Nucl. Phys. B216, 209 (1983).

[12] L. J. Reinders, H. Rubinstein, and S. Yazaki, Phys. Rep. 127, 1 (1985).

[13] H. Shiomi and T. Hatsuda, Nucl. Phys. A594, 294 (1995).

[14] M. C. Birse and B. Krippa, Phys. Lett. B 373, 9 (1996); University of Manchester Report No. MC/TH 96/19.

[15] K. C. Yang, W-Y. P. Hwang, E. M. Henley, and L. S. Kisslinger, Phys. Rev. D 47, 3001 (1993).

[16] J. Gasser and H. Leutwyler, Phys. Rep. 87, 77 (1982); Nucl. Phys. B250, 465 (1985).

[17] G. A. Miller et al., Phys. Rep. 194, 1 (1990).

[18] W-Y. P. Hwang, Ann. Phys. (N.Y.) 174, 247 (1987). 